Biomarkers and Lifestyle (AIBL) study of ageing. A comprehensive metric encapsulating both microscopic changes in fibre density and macroscopic changes in fibre-bundle cross-section (FDC) was obtained for each white matter fixel, and compared across groups using FBA, both at the whole-brain level, and subsequently across specific fibre tracts-of-interest. We further investigated whether changes in FDC were related to amyloid accumulation in MCI patients, by subdividing this group and comparing $A \beta+(n=20)$ and $A \beta-(n=13)$ MCI participants. Results: Whole-brain FBA exhibited significant FDC decreases in AD patients compared to controls, across various fibre tracts (uncinate, inferior fronto-occipital (IFOF), left arcuate fasciculi, splenium and genu) (see figure 1). When statistical analyses were limited to these fibre tracts-of-interest, MCI patients exhibited significant FDC reductions in the bilateral posterior cingulum and right uncinate fasciculus (see figure 2). When MCI patients were subdivided by $\mathrm{A} \beta$ status, only the $\mathrm{A} \beta$ MCI group exhibited significant FDC decrease, and only in the left posterior cingulum. Conclusions: These results suggest substantial reductions in structural connectivity of various WM pathways arise in $\mathrm{AD}$, and exhibit the value of FBA in identifying changes within specific fibre pathways, even in crossing fibre regions. Furthermore, while disruptions to the posterior cingulum and

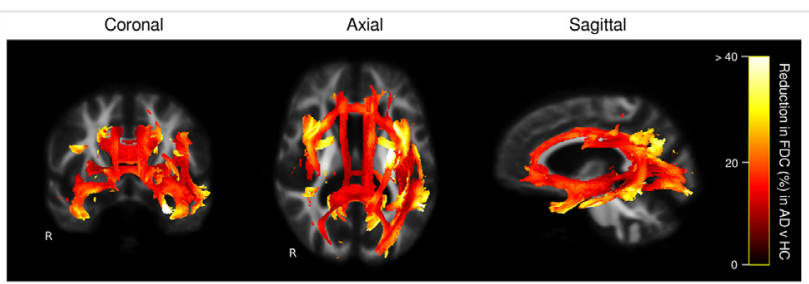

Figure 1. Significant FDC decreases in AD compared to healthy control subjects. Significant reductions in FDC (FWE-corrected p-value $<0.05$ ) upon whole-brain FBA are shown from coronal, axial and sagittal views. Fixels are coloured by percentage decrease in the $\mathrm{AD}$ group compared to healthy controls (HC) as per the scale bar.

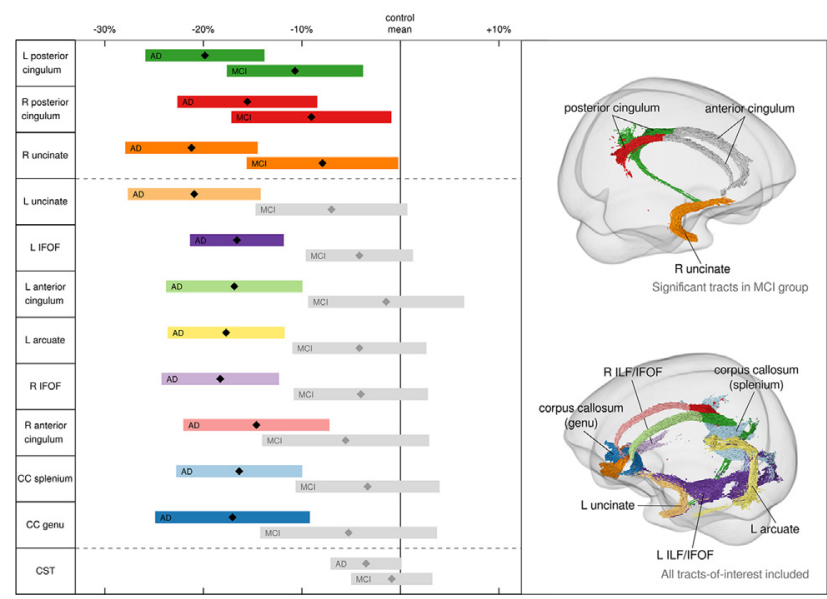

Figure 2. Significant tracts from tract-of-interest analysis.

Left panel: mean FDC (diamonds) and 95\% confidence intervals (bars) for each tract-of-interest displayed for $\mathrm{AD}$ and $\mathrm{MCI}$ groups, displayed as percentage difference from healthy control subjects. Significant tracts $(\mathrm{p}<0.05)$ are displayed above dotted line in colour (bilateral posterior cingulum and right uncinate fasciculus), while non-significant findings shown in grey. The corticospinal tract (CST) is displayed at the bottom for comparison.

Right panel: tracts-of-interest displayed and colour-coded to match left panel. uncinate are likely associated with early cognitive impairment, they do not appear to be associated with high $\mathrm{A} \beta$ accumulation. Further longitudinal studies are necessary to determine any relationship between specific changes in white matter connectivity and progression in $\mathrm{AD}$.

\begin{tabular}{|c|c|c|c|c|}
\hline & $\begin{array}{l}\mathrm{HC} \\
\mathrm{n}=95\end{array}$ & $\begin{array}{l}\text { MCI } \\
\mathrm{n}=33\end{array}$ & $\begin{array}{l}\mathrm{AD} \\
\mathrm{n}=49\end{array}$ & p-value \\
\hline Age & $78.3(7.5)$ & 79.4 (7.6) & $77.4(8.2)$ & 0.51 \\
\hline Males & $45(47.4)$ & $16(48.5)$ & $22(44.9)$ & 0.94 \\
\hline $\begin{array}{l}{ }^{11} \mathrm{C}-\mathrm{PIB} \\
\text { positivity }\end{array}$ & 31 (32.6) & $20(60.6)$ & 49 (100) & $<0.001$ \\
\hline ICV & $1432.6(134.4)$ & 1420.5 (158.6) & $1403.0(137.0)$ & 0.47 \\
\hline
\end{tabular}

\section{IC-P-166 UTILITY OF PERFUSION PET MODELS AS MEASURE OF NEURODEGENERATION IN AN AUTOSOMAL DOMINANT ALZHEIMER'S DISEASE POPULATION: REPORT FROM THE DIAN STUDY}

Nelly Joseph-Mathurin ${ }^{1}$, Yi Su ${ }^{1}$, Tyler Blazey ${ }^{1}$, Mateusz S. Jasielec ${ }^{1}$, Andrei G. Vlassenko ${ }^{1}$, Karl A. Friedrichsen ${ }^{1}$, Brian A. Gordon ${ }^{1}$, Russ C. Hornbeck ${ }^{1}$, Lisa Cash $^{1}$, Beau M. Ances ${ }^{1}$, Marcus E. Raichle ${ }^{1}$, Adam M. Brickman ${ }^{2}$, Virginia Buckles ${ }^{1}$, Nigel J. Cairns ${ }^{1}$,

Carlos Cruchaga ${ }^{1}$, Anne M. Fagan ${ }^{1}$, Alison Goate ${ }^{3}$, Clifford R. Jack, Jr, ${ }^{4}$, Bill E. Klunk ${ }^{5}$, Robert A. Koeppe ${ }^{6}$, Daniel S. Marcus ${ }^{1}$, Richard Mayeux ${ }^{7}$, Eric McDade ${ }^{1}$, Katrina L. Paumier ${ }^{1}$, John M. Ringman ${ }^{8}$, Andrew J. Saykin ${ }^{9}$, Paul M. Thompson ${ }^{8}$, Chengjie Xiong ${ }^{1}$, John C. Morris ${ }^{1}$,

Randall J. Bateman ${ }^{1}$, Tammie L. S. Benzinger ${ }^{1}$ and Dominantly Inherited Alzheimer Network, ${ }^{1}$ Washington University School of Medicine, St. Louis, MO, USA; ${ }^{2}$ Taub Institute for Research on Alzheimer's Disease and the Aging Brain, Columbia University, New York, NY, USA; ${ }^{3}$ Icahn School of Medicine at Mount Sinai, New York, NY, USA; ${ }^{4}$ Mayo Clinic College of Medicine, Rochester, MN, USA; ${ }^{5}$ University of Pittsburgh, Pittsburgh, PA, USA; ${ }^{6}$ University of Michigan, Ann Arbor, MI, USA $;{ }^{7}$ Columbia University, New York, NY, USA $;{ }^{8}$ University of Southern California, Los Angeles, CA, USA; ${ }^{9}$ Indiana University School of Medicine, Indianapolis, IN, USA. Contacte-mail:mathurinn@npg.wustl.edu

Background: 18F-fluorodeoxyglucose (FDG) positron emission tomography (PET) is commonly used to estimate neurodegeneration in Alzheimer's disease (AD). Here, we evaluate the utility of surrogate perfusion measurements obtained from models of perfusion using 11C-Pittsburgh compound B (PiB) to estimate neurodegeneration and to potentially decrease participant burden. Methods: FDG and full dynamic PiB imaging were obtained from 110 participants from the Dominantly Inherited Alzheimer Network (DIAN), including 45 non-carriers (NC, 38.2 \pm 10.1 years) and 65 mutation-carriers (MC, $39.8 \pm 12.0$ years). Two surrogate perfusion measurements were obtained from the PiB scan. The first measurement was SUVRs of early frames of $\mathrm{PiB}$ (ePiB, 1 to $9 \mathrm{~min}$ ) normalized to

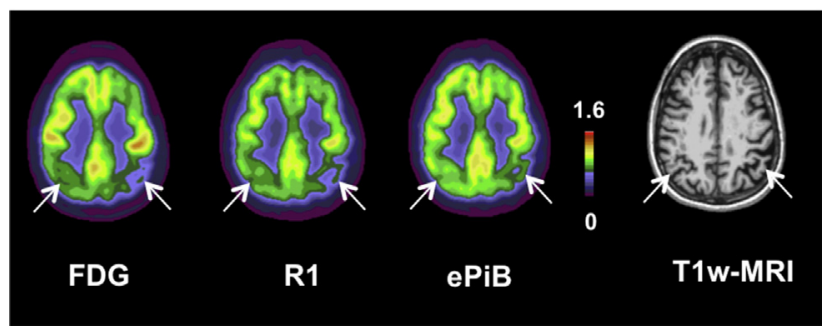




\section{Inferior Parietal}

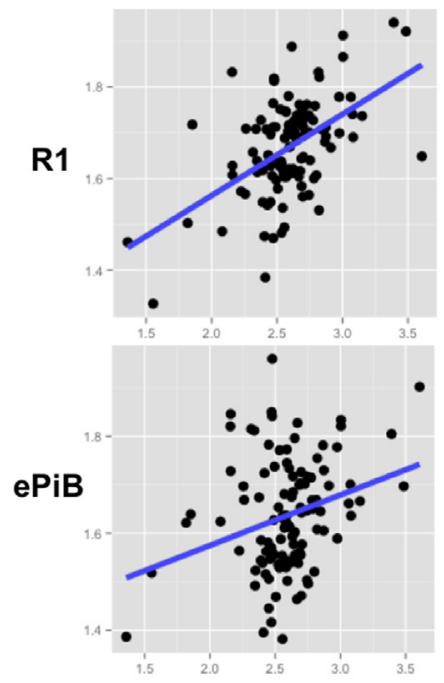

FDG
Precuneus

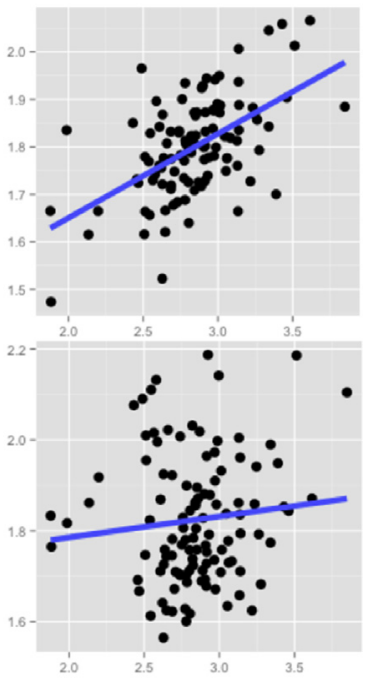

FDG
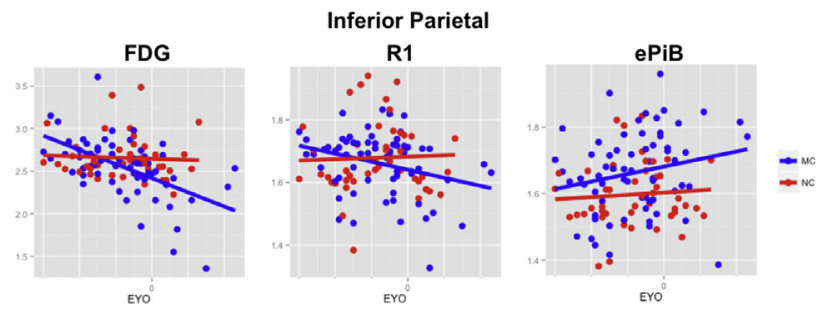

the brainstem. The second measurement was the relative tracer influx rate (R1) obtained from kinetic modeling of PiB data, using brainstem as the reference region. Partial volume correction was applied to take into account the atrophic process. Regional crosssectional analyses were performed to evaluate the correlation between images and estimate the relationship of the imaging biomarkers with estimated time to disease progression based on family history. Nine regions were evaluated, including the precuneus and the inferior parietal cortex, known to be greatly affected by hypometabolism. Results: Metabolism and perfusion images were spatially highly correlated, showing decreased signal in similar regions (Figure 1). Across all participants, the R1 values were better correlated to FDG than ePiB was (e.g. $r=0.52$, $\mathrm{p}<0.0001$ and $\mathrm{r}=0.54, \mathrm{p}<0.0001$ for $\mathrm{R} 1 \mathrm{vs}$. FDG in the inferior parietal and the precuneus, respectively, and $\mathrm{r}=0.28, \mathrm{p}<0.005$ and $\mathrm{r}=0.10$, n.s. for ePiB vs. FDG in the inferior parietal and the precuneus, respectively, Figure 2). Regional R1 values and FDG significantly decreased in the MC vs. NC with estimated-year-to-onset ( $<<0.05$ for the inferior parietal) while ePiB did not decrease but increased instead $(\mathrm{p}<0.05$ for the inferior parietal) (Figure 3). Within the MC, R1 values and FDG significantly decreased with dementia severity $(\mathrm{p}<0.05$ for the inferior parietal) while $\mathrm{ePiB}$ had no relationship with dementia for any regions. Conclusions: Neurodegeneration estimated by R1 may provide a new measure of brain function without added radioactivity. EPiB does not provide good neurodegeneration estimates as it may be contaminated with $\beta$-Amyloid deposition.

\section{IC-P-167 ACROSS-SESSION REPRODUCIBILITY OF AUTOMATIC WHITE MATTER HYPERINTENSITIES SEGMENTATION: A EUROPEAN MULTI-SITE 3T STUDY}

Federica Ribaldi ${ }^{1}$, Moira Marizzoni ${ }^{2}$, Jorge Jovicich ${ }^{3}$, Clarissa Ferrari ${ }^{4}$, Beatriz Bosch $^{5}$, David Bartrés-Faz ${ }^{6}$, Bernhard W. Müller ${ }^{7}$, Jens Wiltfang ${ }^{8}$, Ute Fiedler $^{9}$, Luca Roccatagliata ${ }^{10,11}$, Agnese Picco ${ }^{12}$, Flavio Nobili ${ }^{13}$, Olivier Blin ${ }^{14}$, Stephanie Bombois ${ }^{15}$, Renaud Lopes ${ }^{16}$, Regis Bordet ${ }^{17}$, Julien Sein ${ }^{18}$, Jean-Philippe Ranjeva ${ }^{19}$, Mira Didic ${ }^{20,21}$, Helene GrosDagnac $^{22}$, Pierre Payoux ${ }^{23,24}$, Giada Zoccatelli ${ }^{25}$, Franco Alessandrini ${ }^{25}$, Alberto Beltramello ${ }^{26}$, Nuria Bargalló ${ }^{27}$, Antonio Ferretti ${ }^{28}$,

Massimo Caulo $^{29}$, Marco Aiello $^{30}$, Carlo Cavaliere $^{30}$, Andrea Soricelli $^{30,31}$, Lucilla Parnetti ${ }^{32}$, Robertto Tarducci ${ }^{33}$, Piero Floridi ${ }^{34}$, Magda Tsolaki ${ }^{35}$, Manos Constantinides ${ }^{36}$, Antonios Drevelegas ${ }^{36}$, Paolo Maria Rossini ${ }^{37}$, Camillo Marra $^{38}$, Peter Schonknecht ${ }^{39}$, Tilman Hensch ${ }^{39}$,

Karl-Titus Hoffmann ${ }^{40}$, Joost Kuijer ${ }^{41}$, Pieter Jelle Visser ${ }^{41,42}$,

Frederik Barkhof ${ }^{43}$, Giovanni B. Frisoni ${ }^{44,45},{ }^{1}$ IRCCS Centro San Giovanni di Dio Fatebenefratelli, Brescia, Italy; ${ }^{2}$ IRCCS Istituto Centro San Giovanni di Dio Fatebenefratelli, Brescia, Italy; ${ }^{3}$ University of Trento, Trento, Italy; ${ }^{4}$ Service of Statistics, IRCCS Fatebenefratelli, Brescia, Italy; ${ }^{5}$ Alzheimer's Disease and Other Cognitive Disorders Unit, Neurology Department, IDIBAPS, Hospital Clínic de Barcelona, Barcelona, Spain; ${ }^{6}$ University of Barcelona, Barcelona, Spain; ${ }^{7}$ University of Duisburg-Essen, Essen, Germany; ${ }^{8}$ Department of Psychiatry and Psychotherapy, University Medical Center Göttingen, Göttingen, Germany; ${ }^{9}$ Institutes and Clinics of the University Duisburg-Essen, Essen, Germany; ${ }^{10}$ IRCSS San Martino University Hospital and IST, Genoa, Italy; ${ }^{11}$ Department of Neuroscience, Ophthalmology and Genetics University of Genoa, Genoa, Italy;

${ }^{12}$ Department of Neuroscience, Ophthalmology, Genetics and Mother-Child Health (DINOGMI), University of Genoa, Genoa, Italy;

${ }^{13}$ University of Genoa, Italy, Genoa, Italy; ${ }^{14}$ Aix-Marseille

University-CNRS, Marseille, France; ${ }^{15}$ University of Lille, INSERM U1171, Memory Clinic, F-59000 Lille, Lille, France; ${ }^{16}$ INSERM U1171,

Neuroradiology Department, University Hospital, Lille, France; ${ }^{17}$ Service de Pharmacologie-Hôpital Huriez-CHRU, Lille, France;

${ }^{18}$ CRMBM-CEMEREM, UMR 7339, Aix Marseille University, Marseille, France; ${ }^{19} \mathrm{CIC}$-UPCET, CHU La Timone, AP-HM, UMR CNRS-Universite de la Mediterranee, Marseille, France; ${ }^{20}$ Aix-Marseille Université, Marseille, France; ${ }^{21}$ Service de Neurologie et Neuropsychologie, Marseille, France; ${ }^{22}$ INSERM, Imagerie Cérébrale Et Handicaps Neurologiquies, UMR825, Toulouse, France; ${ }^{23}$ INSERM, Imagerie Cérébrale et Handicaps Neurologiques, Toulouse, France; ${ }^{24}$ Université de Toulouse, Toulouse, France; ${ }^{25}$ Department of Neuroradiology, General Hospital, Verona, Italy; ${ }^{26}$ Department of Neuroradiology, General Hospital, Verona, Italy;

${ }^{27}$ Magnetic Resonance Imaging Core Facility, Institut d'Investigacions Biomèdiques August Pi i Sunyer (IDIBAPS), Barcelona, Spain;

${ }^{28}$ Department of Neuroscience, Imaging and Clinical Sciences, University G. d'Annunzio, Chieti, Italy; ${ }^{29}$ University "G. d'Annunzio" of Chieti, Chieti, Italy; ${ }^{30}$ IRCCS SDN, Naples, Italy; ${ }^{31}$ University of Naples Parthenope, Naples, Italy; ${ }^{32}$ Lab of Clinical Neurochemistry, University of Perugia, Perugia, Italy; ${ }^{33}$ Perugia General Hospital, Medical Physics Unit, Perugia, Italy; ${ }^{34}$ Perugia General Hospital, Neuroradiology Unit, Perugia, Italy; ${ }^{35}$ Aristotle University of Thessaloniki, Thessaloniki, Greece;

${ }^{36}$ Interbalkan Medical Centre of Thessaloniki, Thessaloniki, Greece;

${ }^{37}$ Catholic University, Policlinic Gemelli, Rome, Italy; ${ }^{38}$ Catholic University, Rome, Italy; ${ }^{39}$ Department of Psychiatry and Psychotherapy, University Hospital Leipzig, Germany, Leipzig, Germany; ${ }^{40}$ Department of Neuroradiology, University Hospital Leipzig, Leipzig, Germany; ${ }^{41} \mathrm{VU}$ University Medical Center, Amsterdam, Netherlands; ${ }^{42}$ Maastricht University, Maastricht, Netherlands; ${ }^{43}$ Department of Radiology and Nuclear Medicine, Amsterdam Neuroscience, VU University Medical Center, Amsterdam, Netherlands; ${ }^{44}$ Memory Clinic and LANVIE - Laboratory of Neuroimaging of Aging, University Hospitals and University of Geneva, Geneva, Switzerland; ${ }^{45}$ Laboratory of Alzheimer's Neuroimaging and Epidemiology - LANE, IRCCS Institute - The Saint John of God Clinical Research Centre, Brescia, Italy. Contact e-mail: fribaldi@fatebenefratelli.eu 